\title{
Spin-Wave Emission from Vortex Cores under Static Magnetic Bias Fields
}

Sina Mayr,* Lukáš Flajšman, Simone Finizio, Aleš Hrabec, Markus Weigand, Johannes Förster, Hermann Stoll, Laura J. Heyderman, Michal Urbánek, Sebastian Wintz,* and Jörg Raabe

Cite This: Nano Lett. 2021, 21, 1584-1590

Read Online

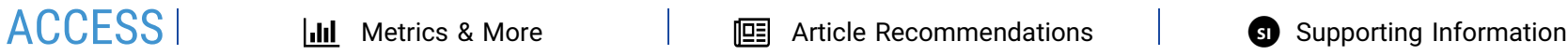

ABSTRACT: We studied the influence of a static in-plane magnetic field on the alternating-field-driven emission of nanoscale spin waves from magnetic vortex cores. Time-resolved scanning transmission X-ray microscopy was used to image spin waves in disk structures of synthetic ferrimagnets and single ferromagnetic layers. For both systems, it was found that an increasing magnetic bias field continuously displaces the wave-emitting vortex core from the center of the disk toward its edge without noticeably altering the spin-wave dispersion relation. In the case of the single-layer disk, an anisotropic lateral expansion of the core occurs at higher magnetic fields, which leads to a directional rather than radial-isotropic emission and propagation of waves. Micromagnetic simulations confirm these findings and further show that focusing effects occur in such systems, depending on the shape of the core and controlled by the static magnetic bias field.
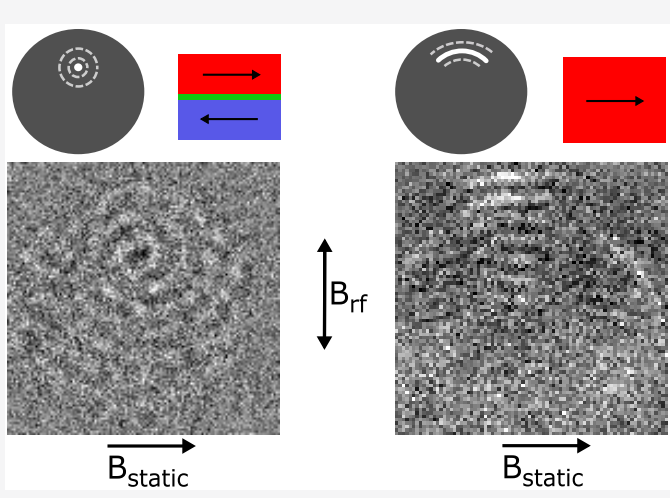

KEYWORDS: magnetization dynamics, magnonics, X-ray microscopy, spin waves, vortex cores

\section{INTRODUCTION}

Spin waves, and their magnon quasiparticles, are the collective elementary excitations of ordered spin systems. In a classical view of a spin wave, magnetic moments precess with a periodic spatial phase shift that determines the wavelength of the excitation (Figure 1a). ${ }^{1,2}$ The research on spin waves ranges from fundamental physics like Bose-Einstein condensates ${ }^{3,4}$ to proposed applications in, for example, logic operations. ${ }^{5,6}$ The utilization of spin waves for devices has two main advantages over present charge-based microelectronic technologies: the first is a low power consumption, because when spin waves are used as signal carriers electric charges are not necessarily displaced, which means that ohmic losses can be prevented. Therefore, the heat dissipation and the power consumption of information processing can be significantly reduced. ${ }^{5}$ The second advantage is the possibility to downscale devices, because the wavelengths of spin-waves are orders of magnitude smaller than those of electromagnetic waves of the same frequency, which typically lies in the gigahertz range. ${ }^{7}$ This property provides a high potential for the development of miniature signal processing devices.

In the context of device miniaturization, the generation of spin waves with wavelengths extending down to the nanoscale therefore becomes important. Typical ways to generate such spin waves include, but are not limited to, patterned microwave antennas through which radio frequency (rf) currents are sent, thus creating Oersted magnetic fields ${ }^{8,9}$ and the exploitation of current-induced spin transfer torques. ${ }^{10-13}$ However, the (a)

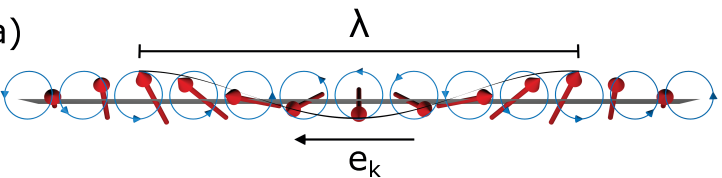

(b)

(c)
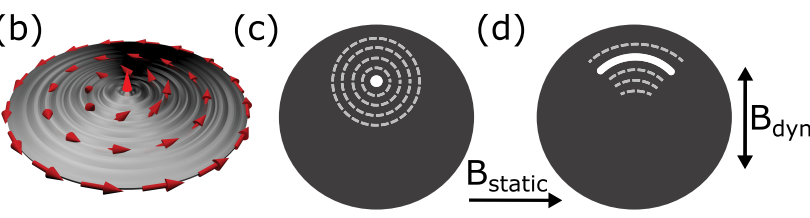

Figure 1. Schematics of spin waves and magnetic vortices. (a) Magnetic moments (red arrows) precess (blue arrows), forming a spin wave with wavelength $\lambda$ that propagates along $e_{\mathrm{k}}$. (b) Alternating field driven oscillation of a magnetic vortex core (red) emitting spin waves in the absence of a static magnetic bias field. Dark red arrows represent the magnetic moments slightly tiling out of the film plane closer to the center, while the grayscale represents the magnetization component in the plane. (c,d) Top view of the response of a vortex to the application of a static magnetic in-plane field (c) without deformation of the perpendicular core (white) leading to a circular emission of spin waves (dashed) and (d) with deformation leading to a directional emission.

Received: September 15, 2020

Revised: December 21, 2020

Published: February 5, 2021 
patterning necessary to realize such field-inhomogeneities at the nanoscale poses a substantial challenge not only in terms of nanofabrication but also for the impedance-matching of such antennas. ${ }^{9}$ Alternatively, natural internal magnetic fieldinhomogeneities such as magnetic domain walls, can be used to generate spin waves in specific textures. ${ }^{14-22}$ In particular, to overcome the minimum wavelength limit given by the characteristic sizes of patterned antennas, it has been shown recently that isotropically propagating spin waves with nanoscale wavelengths $(\sim 100 \mathrm{~nm})$ can be coherently generated by exploiting the dynamics of topological spin textures such as magnetic vortex cores. Driven by alternating magnetic fields and in the absence of magnetic anisotropies, these spin waves propagate away from the vortex core radially (Figure $1 \mathrm{~b}$ ) and are found both in synthetic ferrimagnetic systems as well as in single ferromagnetic layers. ${ }^{18,23-27}$ However, in terms of signal transmission, a directional rather than radial emission and propagation of spin waves would be highly beneficial to reduce the effects of geometrical damping. Such a directional emission can, for example, be achieved in a synthetic ferrimagnet by introducing an intrinsic magnetic anisotropy to the system. Thus, a one-dimensional (1D) magnetic domain wall is formed in addition to the zerodimensional (OD) vortex core. ${ }^{28}$ This additional dimensionality results in a directional plane-wave emission, which is characteristic for spin waves excited by driven magnetic domain walls. ${ }^{22,29,30}$ Despite these examples of such permanently formed domain wall spin-wave emitters, a source for short-wavelength spin waves, whose position and directionality can be continuously manipulated by external stimuli such as a magnetic bias field, is highly desired.

Here, we apply time-resolved scanning transmission X-ray microscopy (TR-STXM) to demonstrate that magnetic vortex cores under the influence of a static magnetic bias field can be used as such manipulable spin-wave emitters. First, we show that one can continuously displace a vortex core from its equilibrium position in the center of a patterned structure in a controlled way while sustaining its driven spin-wave emission (Figure 1c). The extent of this displacement is controlled by the external magnetic bias field and is fully reversible. In contrast to unpatterned thin films, the dispersion relation of these emitted waves in the experimentally observed frequency range is not noticeably affected by the applied magnetic bias field. Furthermore, we show that in a single ferromagnetic layer, a transformation of the vortex core from a point source to an extended object occurs at higher applied fields (Figure 1d). This transformation effectively changes the vortex core from a zero-dimensional (OD) to a one-dimensional (1D) spinwave source. The $1 \mathrm{D}$ character of the emitter leads to a directional emission and propagation of spin waves. The shape of the core depends on the applied magnetic field, making it a manipulable source for planar, propagating spin waves. Finally, we show with micromagnetic simulations that by changing the excitation frequency and the shape of the vortex core, spinwave focusing effects can be achieved.

\section{EXPERIMENTAL METHODS}

For our experiments, two different samples are fabricated. Sample \#1 is a synthetic ferrimagnet ( $\mathrm{SFi}$ ) consisting of a Co/ $\mathrm{Ru} / \mathrm{Ni}_{81} \mathrm{Fe}_{19}$ trilayer where the thickness of the nominally 0.8 $\mathrm{nm}$ thick $\mathrm{Ru}$ is chosen such that it mediates antiferromagnetic interlayer exchange coupling between the $45 \mathrm{~nm}$ thick $\mathrm{Ni}_{81} \mathrm{Fe}_{19}$ (Permalloy, Py) and the $48 \mathrm{~nm}$ thick Co layer. ${ }^{31}$ Sample \#2 consists of a single, nominally $100 \mathrm{~nm}$ thick ferromagnetic (FM) Py film. The films were deposited on $200 \mathrm{~nm}$ thick X-ray transparent $\mathrm{Si}_{3} \mathrm{~N}_{4}$ membranes by direct current magnetron sputtering. In contrast to sample \#1, where all lithography steps were performed on the front side of the $\mathrm{Si}_{3} \mathrm{~N}_{4}$ membrane, the film for sample \#2 was deposited on the back side of the membrane. As the sputter deposition rate is lower onto the depth-confined membrane on the back than onto a flat substrate, the Py film is presumably thinner than the nominal $100 \mathrm{~nm}$. Disk structures of approximately $3 \mu \mathrm{m}$ in diameter were defined by electron beam lithography and consecutively etched out of the continuous films by Ar ion milling. The ground state of these structures are magnetic vortices with an in-plane flux-closure of the magnetization and their central cores oriented perpendicular to the film plane. ${ }^{32,33}$ Because of the antiferromagnetic interlayer coupling, the vortices in the two layers of sample \#1 have opposite in-plane circulations whereas, due to dipolar coupling, their core polarities are parallel. On top of the structures, a conductive, $200 \mathrm{~nm}$ thick, 5 $\mu \mathrm{m}$ wide $\mathrm{Cu}$ microstrip (sample $\# 1$, directly on top) or coplanar waveguide (sample \#2, $\mathrm{Si}_{3} \mathrm{~N}_{4}$ in between) was patterned via electron beam lithography and lift-off processing. Alternating currents on the order of $20 \mathrm{~mA}$ are passed through these conductors to create dynamic magnetic Oersted fields of the order of $\mu_{0} \mathrm{H}=1 \mathrm{mT}$. It is the quasi-uniform in-plane component of these alternating fields that mainly excites the magnetization dynamics in the disks. Both of the sample systems investigated are similar to samples for which the emission of spin waves from vortex cores without the presence of magnetic bias fields has been reported in the past. ${ }^{23,24}$ More details on the sample fabrication can be found in Supporting Information (SI) Section I.

The samples are directly imaged using TR-STXM, which was carried out at the MAXYMUS end station of the BESSY II synchrotron radiation facility of Helmholtz-Zentrum Berlin. For this method, monochromatic X-rays are focused by a Fresnel zone plate to a spot of approximately $25 \mathrm{~nm}$ diameter on the sample and the photons transmitted through the sample are detected by an avalanche photodiode. The sample is raster scanned through the beam to form a two-dimensional image. For time-resolved measurements, a pump-probe technique sensitive to periodic events is used, where the excitation of the sample is synchronized with the probing X-ray flashes from the synchrotron. The time resolution is given by the width of the $\mathrm{X}$-ray bunches and can reach 10 ps with low-alpha optics. ${ }^{24}$ The energy of the photons was tuned either to the Fe $(\sim 709$ $\mathrm{eV})$ or Co $(\sim 781 \mathrm{eV}) \mathrm{L}_{3}$ resonant X-ray absorption edges for selective sensitivity either to the Py or Co layer, respectively. We employ circularly polarized X-rays for gaining magnetic contrast via the X-ray magnetic circular dichroism (XMCD) effect. $^{34}$ The samples were mounted in normal incidence with respect to the incoming X-rays, which results in a sensitivity to the out-of-plane component of the magnetization. Static magnetic fields of up to $40 \mathrm{mT}$ are applied in the sample plane, perpendicular to the dynamic fields that excite the emission of the spin waves. ${ }^{35}$

\section{RESULTS AND DISCUSSION}

When a static in-plane magnetic bias field is applied to the vortex structures in SFi sample \#1, the cores of these vortices are displaced from the disk center congruently in both layers as can be seen for an applied magnetic field of $5 \mathrm{mT}$ in the absolute absorption image in Figure 2a. This means that the 


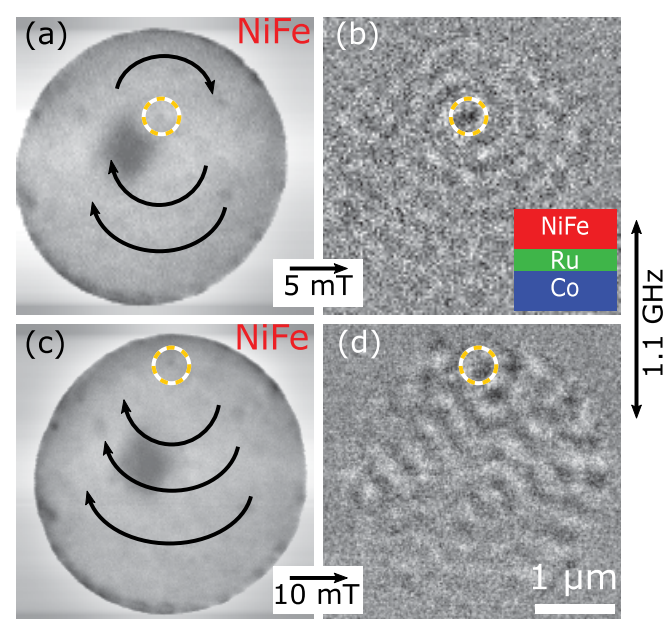

Figure 2. TR-STXM of sample \#1 (SFi), taken with out-of-plane magnetic sensitivity at the $\mathrm{Fe}_{3}$ edge at two different static magnetic fields (5 and $10 \mathrm{mT}$ ). (a,c) Absolute absorption images (time average) showing the position of the vortex core visible as a black dot (corresponding to the magnetization pointing into the disk plane), which is encircled by a yellow dashed line. The dark contrast in the center is topographic. Black arrows represent the in-plane magnetization orientation of the top layer $(\mathrm{NiFe})$ in the disk, where the nonfavorable domain expands. (b,d) Normalized contrast snapshots displaying the radial spin-wave emission from the core for an excitation frequency of $1.1 \mathrm{GHz}$. The inset shows schematics of the SFi trilayer stack.

region of the vortex pair, where the dominant magnetization (in the Co layer) is aligned parallel to the applied field, expands to minimize the Zeeman energy. This behavior is in agreement with previous findings for vortices in single layers ${ }^{36}$ as well as for coupled vortices. ${ }^{37}$ For the strictly antiferromagnetically coupled vortices studied here, in one of the ferromagnetic layers, the unfavorable domain has to expand. For our system, this is the NiFe layer due to its lower magnetic moment per unit area. ${ }^{37}$ When we now apply an rf excitation, spin waves are emitted from the displaced vortex cores. The mechanism behind this emission of short-wavelength spin waves lies in the strongly enhanced local effective magnetic fields and, hence, torques that are present near the vortex core, which couple to the spin-wave continuum. These torques stem from the small oscillating motion that the vortex core performs upon excitation. ${ }^{24}$ We observe a radial emission from the displaced cores in a very similar way as if no static field was applied. An example of such an isotropic emission and radial propagation for an excitation frequency of $1.1 \mathrm{GHz}$ is shown in the normalized snapshot of a time-resolved measurement in Figure 2b (see also SI Movie M1). To form such a set of normalized images, each time frame of the corresponding timeresolved image set is divided by the time average of all frames, therefore highlighting the temporal changes of the magnetization. A congruent emission pattern in both ferromagnetic layers (see SI Section II) confirms that the observed spin waves are layer collective Damon-Eshbach waves, which are also observed when no magnetic bias fields are applied. ${ }^{23}$

Higher magnetic fields $(10 \mathrm{mT})$ lead to a stronger core displacement, further away from the center as depicted in Figure $2 c$, where the vortex core approaches the disk edge. As shown in the normalized view in Figure $2 \mathrm{~d}$, the spin-wave emission remains mainly radial from the source. The visible deviations from the radial emission stem presumably from imperfections in the sample (see also SI Movie M2). ${ }^{38}$ From the normalized images, the wavelengths of the spin waves can be determined to be $348 \pm 12 \mathrm{~nm}$ and $348 \pm 8 \mathrm{~nm}$ for bias fields of $5 \mathrm{mT}$ and $10 \mathrm{mT}$, respectively. This clearly shows that the wavelength and, hence, the dispersion relation of the collective Damon-Eshbach waves is not noticeably affected by the static magnetic bias field in the range that we observe in the experiments. This is because the internal magnetostatic field, as a result of the core displacement, compensates the external magnetic field, and therefore in most of the vortex structure the effective magnetic field remains almost the same as in the nondisplaced case without bias field. For the applied fields of 5 $\mathrm{mT}$ and $10 \mathrm{mT}$, the cores in both layers, Py and Co, shift together without decoupling due to the strong interlayer exchange coupling with a bilinear coupling constant of the order of $\sim-0.3 \mathrm{~mJ} / \mathrm{m}^{2} .{ }^{23}$ When higher magnetic fields are applied, the cores in the two layers can decouple ${ }^{37}$ before the vortices are expelled, and single domain states form.

Sample \#2, consisting only of a single ferromagnetic layer, behaves similarly to the SFi sample for small magnetic fields of below approximately $10 \mathrm{mT}$. These small fields lead to a moderate displacement of the vortex core from the center and
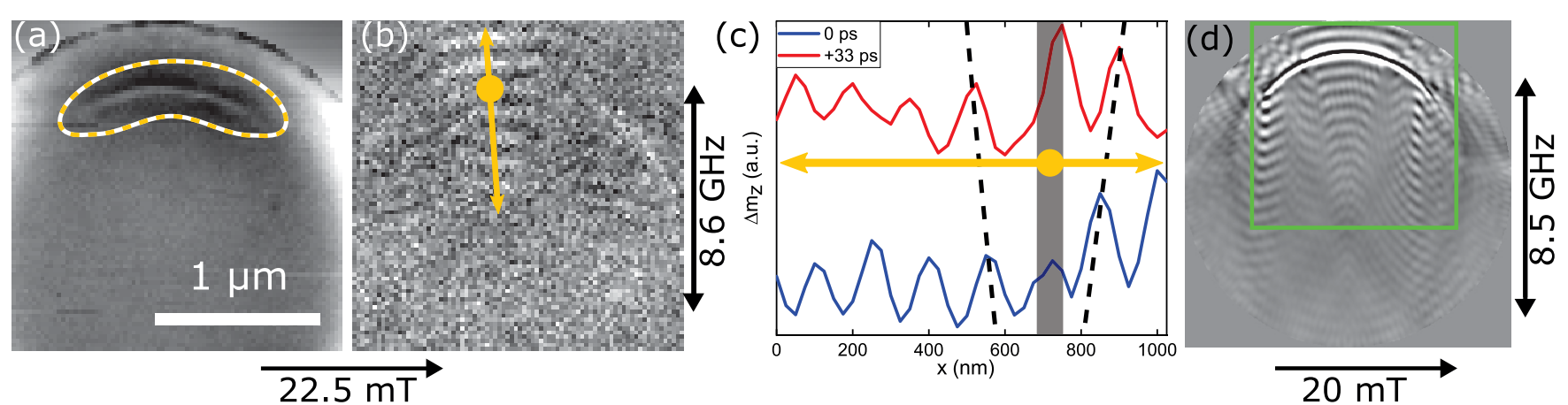

Figure 3. Spin-wave emission from an expanded vortex core. (a,b) TR-STXM with out-of-plane sensitivity displaying the response of the vortex in sample \#2 to an in-plane static magnetic field of $22.5 \mathrm{mT}$ and an alternating field excitation of $8.6 \mathrm{GHz}$ frequency. (a) Absolute absorption image depicting the expanded vortex core displaced from the center (white, encircled by yellow dashes). (b) Normalized snapshot image showing only the temporal changes with respect to the average magnetization, displaying the emitted spin waves. (c) Line profiles along the yellow arrows in (b) at a relative time delay of 33 ps illustrating wave propagation away from the vortex core (gray area) in both directions as supported by the dashed lines. (d) Micromagnetic simulation showing the directional spin-wave emission and subsequent focusing. The green box indicates the region of interest used in the STXM shown in $(a, b)$. 
we observe a radial spin-wave emission from the core. As for the case without a magnetic bias field applied, the emission occurs at higher frequencies than in the SFi, and the observed spin waves correspond to the first higher-order mode of the single-layer Damon-Eshbach geometry with a nonhomogeneous precession profile over the film thickness. ${ }^{23}$ When we apply higher magnetic bias fields, and move the core closer to the edge of the disk, we observe an expansion of the vortex core, ${ }^{39-45}$ as shown in the absolute image in Figure $3 a$. This core expansion is a consequence of the energetically driven tendency of the magnetization to align parallel to the edge of the disk. Thereby, the expanded core resembles a $180^{\circ}$ Bloch domain wall, following the curvature of the rim of the disk.

It should be noted that the expanded core exhibits a nontrivial three-dimensional (3D) structure, where the magnetization at each lateral point is not necessarily fully perpendicular to the plane along the complete thickness crosssection. ${ }^{43}$ In fact, the fully perpendicular fraction of the crosssection connects in a tubular way the separated lateral surface points of fully perpendicular magnetization at the top- and bottom surface of the film (see SI Section III). For the ground state, the combination of core polarity (up or down) and external field direction defines which surface point (top or bottom) is shifted to which end of the expanded core (left or right). In our STXM measurements, where the signal is averaged over the thickness of the film, these slight 3D variations are not visible and we simply consider that the vortex core changes from an effective OD point source to an extended 1D object. ${ }^{39,46-48}$ As a consequence of the expansion of the spin-wave emitter, the emission pattern of the spin waves changes. This emission can be seen in the normalized snapshot of a time-resolved measurement in Figure $3 \mathrm{~b}$ for an excitation frequency of $8.6 \mathrm{GHz}$ (see also SI Movie M2). In contrast to the radial emission that was observed for lower static fields with no pronounced expansion of the core, mostly planar waves propagate away from the expanded core in both directions perpendicular to the long axis of the core. This propagation is highlighted in Figure $3 c$, showing the line profiles along the yellow arrows in Figure $3 \mathrm{~b}$ for two snapshots with a relative time delay of 33 ps. From such line profiles, the extracted wavelength is $146 \mathrm{~nm} \pm 9 \mathrm{~nm}$ for the given frequency of $8.6 \mathrm{GHz}$. A comparison to the case without a static magnetic field, where the wavelength is $143 \mathrm{~nm} \pm 8 \mathrm{~nm}$ (not shown), confirms that, even for the situation of a fieldexpanded vortex core, the wavelength of the spin waves remains almost unaffected by the external field. This is for the same reason of effective field compensation, as already discussed above for the SFi system.

We further observe that, especially for stronger excitations, expanded vortex cores may laterally disintegrate into multiple sections of opposing polarities by presumably forming Bloch points in between the sections (see SI Section IV). ${ }^{39}$ In such a case, the emission of spin waves will become more complex in terms of the emission pattern and phase.

To gain further insight into the spin-wave emission from a 1D expanded vortex core, we performed micromagnetic simulations using MuMax3. ${ }^{49}$ The result of such a simulation is shown as a STXM-comparable snapshot in Figure 3d for an excitation frequency of $8.5 \mathrm{GHz}$ and a magnetic bias field of 20 $\mathrm{mT}$, similar to the experiments. For these simulations, the disk diameter was $3.1 \mu \mathrm{m}$, the thickness was $72 \mathrm{~nm}$, and an initial magnetization of a vortex with anticlockwise circulation and polarity pointing upward was set. This simulated thickness of
$72 \mathrm{~nm}$ is significantly smaller than the nominal experimental thickness of $100 \mathrm{~nm}$, which may result from a reduced sputter deposition rate onto the depth-confined back of the membrane compared to that onto a flat substrate. A cell size of $(3 \times 3 \times$ 3) $\mathrm{nm}^{3}$ was chosen. The static bias field is applied in the film plane with the dynamic excitation field $B_{\text {dyn }}=1 \mathrm{mT}$ perpendicular but also in the film plane, same as in the experiments. We used a saturation magnetization of $M_{\mathrm{s}}=800$ $\mathrm{kA} / \mathrm{m}$, an exchange constant of $A=8 \times 10^{-12} \mathrm{~J} / \mathrm{m}$, a gyromagnetic ratio $\gamma=1.854 \times 10^{11} \mathrm{rad} /(\mathrm{s} \mathrm{T})$ and a Gilbert damping $\alpha=0.01$, which leads to a wavelength of $136 \mathrm{~nm} \pm 5$ $\mathrm{nm}$. Note that the value for the exchange constant $A$ is smaller than what is typically utilized to simulate Py thin films, yet this value is in good agreement with previously investigated Py films of similar thickness. ${ }^{24}$

Besides the central spin-wave emission pattern, the simulations show an equally strong spin-wave emission from the outer parts of the expanded core which occurs only at a much weaker level in the experiments. This difference cannot be conclusively explained at present, but we speculate that the outer parts are more difficult to excite in the experiment due to the influence of material and fabrication imperfections (see SI Section V). We performed micromagnetic simulations at different static magnetic fields and excitation frequencies (see SI Section VI) to confirm the experimental finding that the wavelengths of the spin waves are independent of external bias fields and instead can be tuned by the excitation frequency.

The shape and extent of the deformation of the vortex core can be controlled by the magnetic bias field to influence the emission pattern of the spin waves. The spin waves are not emitted as fully planar waves from the curved core, which leads to a geometrical focusing effect ${ }^{50-53}$ of which indications can be seen in the experiment shown in Figure $3 b$. This is more pronounced in the micromagnetic simulation shown in Figure $3 \mathrm{~d}$. These focusing effects are weaker in the experiments presumably due to an insufficient spin-wave propagation length (see SI Section VII). Therefore, the influence of the magnetic field and excitation frequency are further studied via simulations (see SI Section VI). Here, we see that an increased expansion of the core coincides with a broader spin-wave emission pattern from the central core region, which then leads to a less pronounced focusing of the emitted waves. The extent to which focusing occurs depends on both the applied field bias and the excitation frequency, thereby making the vortex core emitter a controllable lens for short-wavelength spin waves. ${ }^{54-57}$

\section{CONCLUSIONS}

We have demonstrated with direct TR-STXM imaging that a static in-plane magnetic bias field can be used to continuously displace the position of vortex core spin-wave emitters. These emitters are driven by alternating magnetic fields and they act as coherent sources for spin waves with nanoscale wavelengths. In a synthetic ferrimagnetic trilayer structure, it is possible to congruently displace the coupled vortex cores while maintaining their radial spin-wave emission pattern. For a relatively thick single-layer film, higher magnetic fields lead to an expansion of the vortex core from an effective point source to an extended object. This expansion changes the emission pattern of the spin waves from circular to rather planar waves that propagate away from the core in both directions perpendicular to the axis of expansion. We observe that the external magnetic field does not noticeably change the spin- 
wave dispersion relation within the vortex structure as a result of internal field compensation. Furthermore, micromagnetic simulations confirm our experimental findings and predict that for certain static magnetic fields, and thus core shapes, spinwave focusing effects occur. Our results show that magnetic vortex cores can be used as continuously displaceable, reshapeable, and controllable spin-wave sources where a directional emission and the focusing of spin waves is achieved by applying a static magnetic field without the need for additional waveguide patterning.

\section{ASSOCIATED CONTENT}

\section{SI Supporting Information}

The Supporting Information is available free of charge at https://pubs.acs.org/doi/10.1021/acs.nanolett.0c03740.

Detailed information on the sample fabrication, additional measurements on sample \#1, the expansion of the vortex core of sample \#2 at various magnetic bias fields and its influence on the spin-wave emission pattern, the influence of a Bloch point on the emission pattern as well as a comparison of vortex core sizes between experiment and simulation, micromagnetic simulations at different magnetic bias fields and excitation frequencies, and simulations with reduced damping (PDF)

Movie 1: Absolute and normalized contrast of sample \#1 at the $\mathrm{Fe}$ edge at an excitation frequency of $1.1 \mathrm{GHz}$ for static magnetic fields of 5 and $10 \mathrm{mT}$ (AVI)

Movie 2: Absolute and normalized contrast of sample \#2 at an excitation frequency of $8.6 \mathrm{GHz}$ at static magnetic fields of $15,22.5$, and $30 \mathrm{mT}$ (AVI)

Movie 3: Normalized contrast of sample \#1 at the Co (left) and Fe edge (middle and right) at an excitation frequency of $1.1 \mathrm{GHz}$ (left and middle) and $2.1 \mathrm{GHz}$ (right) at a static magnetic field of $5 \mathrm{mT}$ (AVI)

Movie 4: Absolute and normalized contrast of sample \#2 at an excitation frequency of $8.1 \mathrm{GHz}$ at a static magnetic field of $22.5 \mathrm{mT}$ (AVI)

Movie 5: Simulated contrast at excitation frequencies of 8.5 and $9.5 \mathrm{GHz}$, and for static magnetic fields of 20 and $25 \mathrm{mT}$ (AVI)

\section{AUTHOR INFORMATION}

\section{Corresponding Authors}

Sina Mayr - Paul Scherrer Institut, 5232 Villigen PSI, Switzerland; Laboratory for Mesoscopic Systems, Department of Materials, ETH Zurich, 8093 Zurich, Switzerland; 다이.org/0000-0003-4614-9874; Email: sina.mayr@ psi.ch

Sebastian Wintz - Paul Scherrer Institut, 5232 Villigen PSI, Switzerland; Max Planck Institute for Intelligent Systems, 70569 Stuttgart, Germany; Email: wintz@is.mpg.de

\section{Authors}

Lukáš Flajšman - CEITEC BUT, Brno University of Technology, 61200 Brno, Czech Republic; NanoSpin, Department of Applied Physics, Aalto University, FI-00076 Aalto, Finland

Simone Finizio - Paul Scherrer Institut, 5232 Villigen PSI, Switzerland; (1) orcid.org/0000-0002-1792-0626

Aleš Hrabec - Paul Scherrer Institut, 5232 Villigen PSI, Switzerland; Laboratory for Mesoscopic Systems, Department of Materials and Laboratory for Magnetism and Interface Physics, Department of Materials, ETH Zurich, 8093 Zurich, Switzerland

Markus Weigand - Helmholtz-Zentrum Berlin, 12489 Berlin, Germany

Johannes Förster - Max Planck Institute for Intelligent Systems, 70569 Stuttgart, Germany

Hermann Stoll - Max Planck Institute for Intelligent Systems, 70569 Stuttgart, Germany; Institute of Physics, Johannes Gutenberg University Mainz, 55128 Mainz, Germany

Laura J. Heyderman - Paul Scherrer Institut, 5232 Villigen PSI, Switzerland; Laboratory for Mesoscopic Systems, Department of Materials, ETH Zurich, 8093 Zurich, Switzerland

Michal Urbánek - CEITEC BUT, Brno University of Technology, 61200 Brno, Czech Republic; 10 orcid.org/ 0000-0003-0072-2073

Jörg Raabe - Paul Scherrer Institut, 5232 Villigen PSI, Switzerland; 이이.org/0000-0002-2071-6896

Complete contact information is available at:

https://pubs.acs.org/10.1021/acs.nanolett.0c03740

\section{Notes}

The authors declare no competing financial interest.

\section{ACKNOWLEDGMENTS}

We would like to thank Joachim Gräfe, Michael Bechtel and Joe Bailey for their support during the STXM beamtimes, Matthias Kronseder and Christian Back for their help with the sample fabrication, and Roland Mattheis for providing the magnetron sputter deposition of the SFi. The STXM measurements were carried out at the MAXYMUS end station of the BESSY II synchrotron radiation facility of HelmholtzZentrum Berlin. We thank HZB for the allocation of synchrotron radiation beamtime. Additional measurements were performed at the PolLux end station at SLS, PSI, Villigen PSI, Switzerland. The PolLux end station was financed by the German Ministerium für Bildung und Forschung (BMBF) through contracts $05 \mathrm{~K} 16 \mathrm{WED}$ and 05K19WE2. This project received funding from the Swiss National Science Foundation (Grant Agreement 172517). A.H. was funded by the European Union's Horizon 2020 research and innovation programme under Marie Sklodowska-Curie Grant Agreement 794207 (ASIQS).

\section{REFERENCES}

(1) Bloch, F. Zur Theorie des Ferromagnetismus. Eur. Phys. J. A 1930, 61 (3), 206-219.

(2) Gurevich, A.G.; Melkov, G.A. Magnetization Oscillations and Waves; CRC Press: Boca Raton 1996; Vol. 1.

(3) Demokritov, S. O.; Demidov, V. E.; Dzyapko, O.; Melkov, G. A.; Serga, A. A.; Hillebrands, B.; Slavin, A. N. Bose-Einstein condensation of quasi-equilibrium magnons at room temperature under pumping. Nature 2006, 443 (7110), 430-3.

(4) Bozhko, D. A.; Serga, A. A.; Clausen, P.; Vasyuchka, V. I.; Heussner, F.; Melkov, G. A.; Pomyalov, A.; L'vov, V. S.; Hillebrands, B. Supercurrent in a room-temperature Bose-Einstein magnon condensate. Nat. Phys. 2016, 12 (11), 1057-1062.

(5) Kruglyak, V. V.; Demokritov, S. O.; Grundler, D. Magnonics. J. Phys. D: Appl. Phys. 2010, 43 (26), 264001.

(6) Neusser, S.; Grundler, D. Magnonics: Spin Waves on the Nanoscale. Adv. Mater. 2009, 21 (28), 2927-2932.

(7) Chumak, A. V.; Vasyuchka, V. I.; Serga, A. A.; Hillebrands, B. Magnon spintronics. Nat. Phys. 2015, 11 (6), 453-461. 
(8) Wagner, K.; Kákay, A.; Schultheiss, K.; Henschke, A.; Sebastian, T.; Schultheiss, H. Magnetic domain walls as reconfigurable spin-wave nanochannels. Nat. Nanotechnol. 2016, 11 (5), 432-436.

(9) Haldar, A.; Kumar, D.; Adeyeye, A. O. A reconfigurable waveguide for energy-efficient transmission and local manipulation of information in a nanomagnetic device. Nat. Nanotechnol. 2016, 11 (5), 437-443.

(10) Demidov, V. E.; Urazhdin, S.; Demokritov, S. O. Direct observation and mapping of spin waves emitted by spin-torque nanooscillators. Nat. Mater. 2010, 9 (12), 984-988.

(11) Madami, M.; Bonetti, S.; Consolo, G.; Tacchi, S.; Carlotti, G.; Gubbiotti, G.; Mancoff, F. B.; Yar, M. A.; Åkerman, J. Direct observation of a propagating spin wave induced by spin-transfer torque. Nat. Nanotechnol. 2011, 6, 635.

(12) Urazhdin, S.; Demidov, V. E.; Ulrichs, H.; Kendziorczyk, T.; Kuhn, T.; Leuthold, J.; Wilde, G.; Demokritov, S. O. Nanomagnonic devices based on the spin-transfer torque. Nat. Nanotechnol. 2014, 9 (7), 509-13.

(13) Bonetti, S.; Kukreja, R.; Chen, Z.; Spoddig, D.; Ollefs, K.; Schoppner, C.; Meckenstock, R.; Ney, A.; Pinto, J.; Houanche, R.; Frisch, J.; Stohr, J.; Durr, H. A.; Ohldag, H. Microwave soft x-ray microscopy for nanoscale magnetization dynamics in the $5-10 \mathrm{GHz}$ frequency range. Rev. Sci. Instrum. 2015, 86 (9), 093703.

(14) Van de Wiele, B.; Hämäläinen, S. J.; Baláž, P.; Montoncello, F.; van Dijken, S. Tunable short-wavelength spin wave excitation from pinned magnetic domain walls. Sci. Rep. 2016, 6 (1), 21330.

(15) Whitehead, N. J.; Horsley, S. A. R.; Philbin, T. G.; Kuchko, A. N.; Kruglyak, V. V. Theory of linear spin wave emission from a Bloch domain wall. Phys. Rev. B: Condens. Matter Mater. Phys. 2017, 96 (6), 064415.

(16) Holländer, R. B.; Müller, C.; Schmalz, J.; Gerken, M.; McCord, J. Magnetic domain walls as broadband spin wave and elastic magnetisation wave emitters. Sci. Rep. 2018, 8 (1), 13871.

(17) Hämäläinen, S. J.; Brandl, F.; Franke, K. J. A.; Grundler, D.; van Dijken, S. Tunable Short-Wavelength Spin-Wave Emission and Confinement in Anisotropy-Modulated Multiferroic Heterostructures. Phys. Rev. Appl. 2017, 8 (1), 014020.

(18) Davies, C. S.; Poimanov, V. D.; Kruglyak, V. V. Mapping the magnonic landscape in patterned magnetic structures. Phys. Rev. B: Condens. Matter Mater. Phys. 2017, 96 (9), 094430.

(19) Mozooni, B.; McCord, J. Direct observation of closure domain wall mediated spin waves. Appl. Phys. Lett. 2015, 107 (4), 042402.

(20) Yu, H.; d'Allivy Kelly, O.; Cros, V.; Bernard, R.; Bortolotti, P.; Anane, A.; Brandl, F.; Heimbach, F.; Grundler, D. Approaching soft $\mathrm{X}$-ray wavelengths in nanomagnet-based microwave technology. Nat. Commun. 2016, 7 (1), 11255.

(21) Voto, M.; Lopez-Diaz, L.; Martinez, E. Pinned domain wall oscillator as a tuneable direct current spin wave emitter. Sci. Rep. 2017, 7 (1), 13559.

(22) Hermsdoerfer, S. J.; Schultheiss, H.; Rausch, C.; Schäfer, S.; Leven, B.; Kim, S.-K.; Hillebrands, B. A spin-wave frequency doubler by domain wall oscillation. Appl. Phys. Lett. 2009, 94 (22), 223510.

(23) Wintz, S.; Tiberkevich, V.; Weigand, M.; Raabe, J.; Lindner, J.; Erbe, A.; Slavin, A.; Fassbender, J. Magnetic vortex cores as tunable spin-wave emitters. Nat. Nanotechnol. 2016, 11 (11), 948-953.

(24) Dieterle, G.; Förster, J.; Stoll, H.; Semisalova, A. S.; Finizio, S.; Gangwar, A.; Weigand, M.; Noske, M.; Fähnle, M.; Bykova, I.; Gräfe, J.; Bozhko, D. A.; Musiienko-Shmarova, H. Y.; Tiberkevich, V.; Slavin, A. N.; Back, C. H.; Raabe, J.; Schütz, G.; Wintz, S. Coherent Excitation of Heterosymmetric Spin Waves with Ultrashort Wavelengths. Phys. Rev. Lett. 2019, 122 (11), 117202.

(25) Behncke, C.; Adolff, C. F.; Lenzing, N.; Hänze, M.; Schulte, B.; Weigand, M.; Schütz, G.; Meier, G. Spin-wave interference in magnetic vortex stacks. Communications Physics 2018, 1 (1), 50.

(26) Osuna Ruiz, D.; Parra, E. B.; Bukin, N.; Heath, M.; Lara, A.; Aliev, F. G.; Hibbins, A. P.; Ogrin, F. Y. Dynamics of spiral spin waves in magnetic nanopatches: Influence of thickness and shape. Phys. Rev. B: Condens. Matter Mater. Phys. 2019, 100 (21), 214437.
(27) Chang, L.-J.; Chen, J.; Qu, D.; Tsai, L.-Z.; Liu, Y.-F.; Kao, M.Y.; Liang, J.-Z.; Wu, T.-S.; Chuang, T.-M.; Yu, H.; Lee, S.-F. Spin Wave Injection and Propagation in a Magnetic Nanochannel from a Vortex Core. Nano Lett. 2020, 20 (5), 3140-3146.

(28) Sluka, V.; Schneider, T.; Gallardo, R. A.; Kákay, A.; Weigand, M.; Warnatz, T.; Mattheis, R.; Roldán-Molina, A.; Landeros, P.; Tiberkevich, V.; Slavin, A.; Schütz, G.; Erbe, A.; Deac, A.; Lindner, J.; Raabe, J.; Fassbender, J.; Wintz, S. Emission and propagation of 1D and $2 \mathrm{D}$ spin waves with nanoscale wavelengths in anisotropic spin textures. Nat. Nanotechnol. 2019, 14 (4), 328-333.

(29) Albisetti, E.; Petti, D.; Sala, G.; Silvani, R.; Tacchi, S.; Finizio, S.; Wintz, S.; Calò, A.; Zheng, X.; Raabe, J.; Riedo, E.; Bertacco, R. Nanoscale spin-wave circuits based on engineered reconfigurable spin-textures. Communications Physics 2018, 1 (1), 56.

(30) Albisetti, E.; Tacchi, S.; Silvani, R.; Scaramuzzi, G.; Finizio, S.; Wintz, S.; Rinaldi, C.; Cantoni, M.; Raabe, J.; Carlotti, G.; Bertacco, R.; Riedo, E.; Petti, D. Optically Inspired Nanomagnonics with Nonreciprocal Spin Waves in Synthetic Antiferromagnets. Adv. Mater. 2020, 32 (9), 1906439.

(31) Grünberg, P.; Schreiber, R.; Pang, Y.; Brodsky, M. B.; Sowers, H. Layered Magnetic Structures: Evidence for Antiferromagnetic Coupling of Fe Layers across Cr Interlayers. Phys. Rev. Lett. 1986, 57 (19), 2442-2445.

(32) Raabe, J.; Pulwey, R.; Sattler, R.; Schweinböck, T.; Zweck, J.; Weiss, D. Magnetization pattern of ferromagnetic nanodisks. J. Appl. Phys. 2000, 88 (7), 4437-4439.

(33) Shinjo, T.; Okuno, T.; Hassdorf, R.; Shigeto, K.; Ono, T. Magnetic Vortex Core Observation in Circular Dots of Permalloy. Science 2000, 289 (5481), 930-932.

(34) Schutz, G.; Wagner, W.; Wilhelm, W.; Kienle, P.; Zeller, R.; Frahm, R.; Materlik, G. Absorption of circularly polarized x rays in iron. Phys. Rev. Lett. 1987, 58 (7), 737-740.

(35) Nolle, D.; Weigand, M.; Audehm, P.; Goering, E.; Wiesemann, U.; Wolter, C.; Nolle, E.; Schütz, G. Note: Unique characterization possibilities in the ultra high vacuum scanning transmission $\mathrm{x}$-ray microscope (UHV-STXM) "MAXYMUS" using a rotatable permanent magnetic field up to 0.22 T. Rev. Sci. Instrum. 2012, 83 (4), 046112.

(36) Guslienko, K. Y.; Novosad, V.; Otani, Y.; Shima, H.; Fukamichi, $K$. Magnetization reversal due to vortex nucleation, displacement, and annihilation in submicron ferromagnetic dot arrays. Phys. Rev. B: Condens. Matter Mater. Phys. 2001, 65 (2), 024414.

(37) Wintz, S.; Bunce, C.; Banholzer, A.; Körner, M.; Strache, T.; Mattheis, R.; McCord, J.; Raabe, J.; Quitmann, C.; Erbe, A.; Fassbender, J. Interlayer-coupled spin vortex pairs and their response to external magnetic fields. Phys. Rev. B: Condens. Matter Mater. Phys. 2012, 85 (22), 224420.

(38) Abeed, M. A.; Sahoo, S.; Winters, D.; Barman, A.; Bandyopadhyay, $\mathrm{S}$. The effect of material defects on resonant spin wave modes in a nanomagnet. Sci. Rep. 2019, 9 (1), 16635.

(39) Im, M.-Y.; Han, H.-S.; Jung, M.-S.; Yu, Y.-S.; Lee, S.; Yoon, S.; Chao, W.; Fischer, P.; Hong, J.-I.; Lee, K.-S. Dynamics of the Bloch point in an asymmetric permalloy disk. Nat. Commun. 2019, 10 (1), 593.

(40) Aliev, F. G.; Sierra, J. F.; Awad, A. A.; Kakazei, G. N.; Han, D.S.; Kim, S.-K.; Metlushko, V.; Ilic, B.; Guslienko, K. Y. Spin waves in circular soft magnetic dots at the crossover between vortex and single domain state. Phys. Rev. B: Condens. Matter Mater. Phys. 2009, 79 (17), 174433.

(41) Vaňatka, M.; Urbánek, M.; Jíra, R.; Flajšman, L.; Dhankhar, M.; Im, M.-Y.; Michaliáka, J.; Uhlí̌, V.; Ćikola, T. Magnetic vortex nucleation modes in static magnetic fields. AIP Adv. 2017, 7 (10), 105103.

(42) Kuepper, K.; Bischoff, L.; Akhmadaliev, C.; Fassbender, J.; Stoll, H.; Chou, K. W.; Puzic, A.; Fauth, K.; Dolgos, D.; Schütz, G.; Van Waeyenberge, B.; Tyliszczak, T.; Neudecker, I.; Woltersdorf, G.; Back, C. H. Vortex dynamics in Permalloy disks with artificial defects: Suppression of the gyrotropic mode. Appl. Phys. Lett. 2007, 90 (6), 062506. 
(43) Graf, J.; Pfeifer, H.; Marquardt, F.; Viola Kusminskiy, S. Cavity optomagnonics with magnetic textures: Coupling a magnetic vortex to light. Phys. Rev. B: Condens. Matter Mater. Phys. 2018, 98 (24), 241406.

(44) Bryant, P.; Suhl, H. Magnetization and domain structure of cylinders and spheres in subsaturating fields. Appl. Phys. Lett. 1989, 54 (1), 78-80.

(45) Pulecio, J. F.; Pollard, S. D.; Warnicke, P.; Arena, D. A.; Zhu, Y. Symmetry breaking of magnetic vortices before annihilation. Appl. Phys. Lett. 2014, 105 (13), 132403.

(46) Jubert, P.-O.; Toussaint, J.-C.; Fruchart, O.; Meyer, C.; Samson, Y. Flux-closure-domain states and demagnetizing energy determination in sub-micron size magnetic dots. Europhys. Lett. 2003, 63 (1), 132-138.

(47) Masseboeuf, A.; Fruchart, O.; Toussaint, J. C.; Kritsikis, E.; Buda-Prejbeanu, L.; Cheynis, F.; Bayle-Guillemaud, P.; Marty, A. Dimensionality Crossover in Magnetism: From Domain Walls (2D) to Vortices (1D). Phys. Rev. Lett. 2010, 104 (12), 127204.

(48) Hertel, R.; Kronmüller, H. Computation of the magnetic domain structure in bulk permalloy. Phys. Rev. B: Condens. Matter Mater. Phys. 1999, 60 (10), 7366-7378.

(49) Vansteenkiste, A.; Leliaert, J.; Dvornik, M.; Helsen, M.; GarciaSanchez, F.; van Waeyenberge, B. The design and verification of MuMax3. AIP Adv. 2014, 4 (10), 107133.

(50) Demidov, V. E.; Demokritov, S. O.; Rott, K.; Krzysteczko, P.; Reiss, G. Self-focusing of spin waves in Permalloy microstripes. Appl. Phys. Lett. 2007, 91 (25), 252504.

(51) Schneider, T.; Serga, A. A.; Chumak, A. V.; Sandweg, C. W.; Trudel, S.; Wolff, S.; Kostylev, M. P.; Tiberkevich, V. S.; Slavin, A. N.; Hillebrands, B. Nondiffractive subwavelength wave beams in a medium with externally controlled anisotropy. Phys. Rev. Lett. 2010, 104 (19), 197203.

(52) Kim, J. V.; Stamps, R. L.; Camley, R. E. Spin Wave Power Flow and Caustics in Ultrathin Ferromagnets with the DzyaloshinskiiMoriya Interaction. Phys. Rev. Lett. 2016, 117 (19), 197204.

(53) Veerakumar, V.; Camley, R. E. Magnon focusing in thin ferromagnetic films. Phys. Rev. B: Condens. Matter Mater. Phys. 2006, 74 (21), 214401.

(54) Whitehead, N. J.; Horsley, S. A. R.; Philbin, T. G.; Kruglyak, V. V. A Luneburg lens for spin waves. Appl. Phys. Lett. 2018, 113 (21), 212404.

(55) Dzyapko, O.; Borisenko, I. V.; Demidov, V. E.; Pernice, W.; Demokritov, S. O. Reconfigurable heat-induced spin wave lenses. Appl. Phys. Lett. 2016, 109 (23), 232407.

(56) Toedt, J.-N.; Mundkowski, M.; Heitmann, D.; Mendach, S.; Hansen, W. Design and construction of a spin-wave lens. Sci. Rep. 2016, 6 (1), 33169.

(57) Gräfe, J.; Gruszecki, P.; Zelent, M.; Decker, M.; Keskinbora, K.; Noske, M.; Gawronski, P.; Stoll, H.; Weigand, M.; Krawczyk, M.; Back, C. H.; Goering, E. J.; Schütz, G. Direct observation of spin-wave focusing by a Fresnel lens. Phys. Rev. B: Condens. Matter Mater. Phys. 2020, 102 (2), 024420. 